\title{
Perspectives sur l'indépendance de la justice arbitrale en Afrique subsaharienne. Les influences croisées entre la Cour Commune de Justice et d'Arbitrage (CCJA) et l'East African Community's Court of Justice (EACJ).
}

\author{
Christian Bahati Bahalaokwibuye*
}

\section{Résumée}

Le présent article pose les perspectives sur les influences opportunes et mutuelles des systèmes arbitraux de la CCJA et l'EACJ dans la promotion de l'indépendance des arbitres. Il montre que, tout comme pour l'EAC, l'abitrage figure déjà dans le Traité de l'OHADA comme le mode de règlement des différends en vue d'améliorer le climat des investissements sur les territoires des Etats parties. La CCJA n'est pas un tribunal arb itral. Son intervention est placée en aval, comme juge de contrôle de la sentence bien qu'elle ne se contente pas d'un contrôle minimal. L'EACJ, qui a retenu l'option du cumul des fonctions de ses juges avec celles d'arbtres, pourra aussi, dans la moindre mesure, se contenter d'administrer les arbitrages ouverts conformément à son Règlement d'arbitrage. De ce point de vue, le système EACJ sera en parfait accord avec l'esprit du principe d'indépendancel impartialité.

En cette matière, nous pensons que la pratique de la CCJA ne manquera pas d'inspirer utilement des réformes du système d'arbitrage de l'EACJ, et qui révèle de plus en plus une prise de conscience certaine d'une croissante indépendance de ses arbitres en ayant opté pour la gratuité de l'arbitrage. Comme le Centre d'arbitrage de la CCJA est attaché à la Cour et que cette dernière est dotée d'une autonomie financière, il est temps de prendre des mesures de réduction de coût, non pas totalement analogues à celles de l'EACJ, à travers lesquelles on dispensera, par exemple les ressortissants de l'espace OHADA et les investisseurs étrangers, des frais administratifs de l'arbitrage. Il en résulterait un arbitrage sans influences, alternative crédible à la justice étatique qui affiche la lanterne rouge et facile d'accès aux justiciables impécunieux, qui rendra des sentences impartiales et légitimes.

\footnotetext{
Abstract

This article presents some perspectives on the timely and mutual influences between the CCJA's and the EACJ's arbitral systems, in promoting the independence of arbitrators. It shows that, like in the EAC's Treaty, arbitration is enshrined in the OHADA Treaty as the

* Docteur en Droit de l'Université Libre de Bruxelles (VUB), Professeur de droit des affaires à l'Université Catholique de Bukavu, Avocat au Barreau près la Cour d'appel de Bukavu (Partner Mercalex).
} 
dispute settlement mechanism tailored to better the investment climate in the States Parties. CCJA is not an arbitral tribunal. It intervenes at the end of arbitral proceedings, simply to control of the arbitral award - although it does not limit itself to a formal control. The EACJ, despite its choice of combining the functions of its judges with those of arbitrators, limits itself to the administration of arbitrations initiated in accordance with its Arbitration Rules, so as to be in line with the spirit of the principle of independence / impartiality.

In that regard, we believe that reforms in the context of CCJA should be inspired by EACJ's arbitration system, and which seems to be more adapted to promoting the independence of arbitrators, by providing for free arbitration. Since the CCJA's Arbitration Center is attached to the Court and the Court has financial autonomy, it is time to take cost reduction measures, although not completely similar to those of the EACJ, but intended to ensure that, for instance, nationals of the OHADA area and foreign investors exempted from administrative costs of arbitration procedure. This may lead to an arbitration without influence; a credible alternative to State justice that displays the red lantern and easy access to impecunious parties, and which will lead to impartial and legitimate awards.

\section{Introduction}

D'entrée de jeu, il faut souligner que l'Organisation pour l'Harmonisation en Afrique du Droit des Affaires (OHADA) et l'East African Community (EAC) sont deux organisations internationales qui ont vocation à contribuer à la promotion des échanges commerciaux internationaux en Afrique subsaharienne ${ }^{1}$.

Il n'est plus admis aucun doute que la mondialisation des échanges a entrainé avec elle la globalisation du droit, amenant à s'interroger sur la nécessité de rapprochement des différentes ces deux initiatives régionales. L'OHADA tout comme l'EAC cherchent à contribuer à l'intégration respectivement juridique et économique en Afrique. Les Etats de l'OHADA se proposent de favoriser l'institution d'une Communauté économique africaine, « en vue d'accomplir de nouveaux progrès sur la voie de l'unité africaine $»^{2}$. Les enjeux politicoéconomiques qui ont cours sur le continent et qui tendent à la création d'une zone de libreéchange à l'échelle continentale, plaident en faveur de ce rapprochement. L'intégration juridique de l'OHADA servirait ainsi de complément utile à l'intégration économique très

1 Plus d'un lecteur peut être frappé de l'étonnement en lisant l'intitulé de cette étude qui tente le rapprochement des avantages comparatifs du système arbitral de l'OHADA et celui de l'EAC. İl est vrai que l'OHADA reste encore moins familère de nombreux juristes anglosaxons surtout ceux de l'EAC. Au regard des objectifs des traités portant leur création, de la position géographique des Etats qui le composent, de leurs actions et réalisations concrètes, de leur organisation institutionnelle ou encore de la désintégration de leurs droits matériels dérivant de leurs traités fondateurs, l'OHADA et l'EAC sont, a priori, faites pour ne pas converger. Mais l'association entre les deux organisations n'est pas contre nature, comme nous allons le montrer plus loin.

2 Cf. Préambule du Traité créant l'Organisation pour l'Harmonisation en Afrique du Droit des Affaires (OHADA) du 17 octobre 1993 tel que révisé le 17 octobre 2008. 
avancée de l'EAC. D'autres enjeux géopolitiques inavoués serviraient de pont entre l'EAC et l'OHADA qu'on ne peut citer ici ${ }^{3}$.

İl convient donc, à ce stade de l'analyse, de tenter une comparaison qui permettrait alors de relever les éléments de cousinage entre le processus d'intégration de l'OHADA et celui de l'EAC. Ce débat sur les emprunts possibles entre l'arbitrage EACJ et CCJAoffre une nouvelle occasion de s'exprimer et de vulgariser des valeurs éthiques inaliénables à observer dans l'arbitrage. Ce débat est révelateur de la nécessité de dialogue, de rencontre entre ordres juridiques arbitraux en vue d'une justice arbitrale indépendante et moins ruineuse ${ }^{4}$.

Laissant de côté les aspects substantiels ${ }^{5}$, on cherchera à se rapprocher de la thématique générale des assises de Mombassa, axées sur "Les défis actuels pour une justice indépendante et efficace en Afrique de l'Est', en ne concentrant nos propos que sur l'organisation judiciaire de l'EAC et de l'OHADA.

À l'étude du paysage judiciaire dans l'espace OHADA, on peut observer que la justice est l'un des grands corps malades de ses pays. Ainsi, les défis de l'indépendance et l'efficacité de la justice ont été placés au cœur de l'insécurité judiciaire dans l'espace OHADA. Cette dernière compte enrayer l'insécurité judiciaire en privilégiant le recours aux modes alternatifs de règlement des conflits (MARC). De ces MARCS, l'arbitrage emporte beaucoup plus les faveurs de l'EAC et de l'OHADA. L'arbitrage y prend de plus en plus d'ampleur au sein de l'EACJ et de la CCJA.

Il se trouve qu'aussi bien l'EACJ et que la CCJA sont des institutions dotées des fonctions hybrides à la fois juridictionnelles et arbitrales et qui contribuent de ce fait à l'indépendance de la justice commerciale des différents Etats membres. La compétence de la CCJA se decline en matière contentieuse, consultative $e^{6}$ et arbitrale. La Cour de justice de

3 On peut penser tout de suite à l'appartenance commune de certains Etats membres de l'OHADA et ceux de l'EAC à la Conférence internationale sur la région des Grands Lacs africains (CIRGL).

4 Cette question est d'une brulante actualité placée, par exemple, dans le contexte de l'Accord CETA entre l'Union européenne et le Canada. La réforme de l'arbitrage qui y est proposé ne semble pas garantir une jusitce indépendante, impartiale et accessible à tous.

5 İl y a lieu d'établir des comparaisons entre ces deux systèmes d'intégration en se basant sur des aspects purement substantiels à l'instar des règles relatives à l'établissement des entreprises (capital social minimum exigé pour bénéficier du régime de Codes des investissement qui n'est pas encore uniformisé dans l'EAC alors que dans l'espace OHADA on veut promouvoir la création des entreprises en y prévoyant un régime uniforme qui varie suivant les formes des sociétés, le temps à prendre pour créer une entreprise dans l'espace OHADA ou dans l'EAC, le droit des sociétés coopératives de l'OHADA comparé à celui de l'EAC, etc.)

6 En sa qualité d'organe juridictionnel, l'article 14-2 du Traité révisé donne la compétence à la CCJA d'être consultée sur toute question relative à l'interprétation et à l'application du Traité, des Règlements, des Actes uniformes et des Décisions. Cet avis consultatif peut même être sollicité par une juridiction nationale de fond saisie d'une question mettant en jeu l'application des Actes uniformes. La CCJA exerce également une fonction contentieuse en examinant le recours qui lui sont soumis et une fonction consultative par laquelle elle émet des avis sur des demandes qui lui sont adressées en 
l'EAC est également pourvue de cette triple compétence contentieuse ${ }^{7}$, consultative ${ }^{8}$ et arbitrale?.

Dans cette optique, la « modernisation ${ }^{10}$ de l'arbitrage est devenue un moyen de sécurisation des investissements internationaux et nationaux au sein des espaces OHADA et $\mathrm{EAC}^{11}$. Les deux juridictions ne peuvent s'atteler à rendre des décisions en matière arbitrales sans se préoccuper de leur exécution ultérieure. Dans ce processus de circulation des sentences arbitrales, une décision rendue sous les auspices de la CCJA peut atteindre des territoires du ressort exclusif de l'EACJ et vice versa ${ }^{12}$.

dehors de toute instance judiciaire. Dans sa compétence contentieuse, aux termes de l'article 14 (3 et 4) du Traité et art. 28-1 du Règlement de procédure de la CCJA, la CCJA est une Cour de cassation communautaire unique pour toute question relative à l'application ou à l'interprétation du Traité et de actes uniformes. Contrairement à la CJEAC qui juge des questions communautaires en instance et en appel, la CCJA n'intervient qu'en cassation. Mais, la CCJA ne se limite pas à casser l'arrêt attaqué, elle évoque en connaissant du fond de l'affaire. Cela donne une empreinte singulière à sa procédure de cassation. La CCJA a également une compétence pour connaître directement de tout litige opposant l'OHADA à l'un de ses fonctionnaires ou à l'un de ses agents. Mais cela ne peut qu'après échec d'un recours préalable auprès de l'autorité hiérarchique compétente. Cf. art. 84-85 du Règlement portant statut des fonctionnaires; art. 55-56 du Règlement portant statut du personnel non fonctionnaire. Du point de vue des effets de ses décisions, aux termes de l'article 20 du Traité de l'OHADA « les arrêts de la Cour Commune de Justice et d'Arbitrage » ont l'autorité de la chose jugée et la force exécutoire ". Ses décisions sont définitives sous réserve de tierce opposition ou de révision, et donnent lieu à l'exécution forcée sur les territoires de tous les Etats parties. En ce sens, ce décisions de la CCJA sont dispensées de la procédure d'exequatur. Toutefois, l'article 46 du Règlement de procédure renseigne que la formule exécutoire est apposée, sans autre contrôle que celui de la vérification de l'authenticité du titre, par l'autorité nationale que le Gouvernement de chacun des Etats parties désignera à cet effet et dont il donnera connaissance à la Cour. Lire aussi, le Règlement d'arbitrage de la CCJA, art. 47 et 49.

7 Cf. Traité de l'EAC, art. 27 (1).

8 Traité de l'EAC, art. 36.

9 Traité de l'EAC, art. 32.

10 İl s'agit bien d'une modernisation mais qui ne va pas revolutionner les moeurs en Afrique subsaharienne. Car, dans le contexte sociologique et juridique africain, l'arbitrage moderne semble d'ailleurs entretenir de parenté avec les procédures juridictionnelles coutumières pratiquées en Afrique précoloniale. Le règlement des litiges privés, bien que moins structuré et pratiqué sans confidentialité, était beaucoup plus marqué par le recours à des modes non jurictionnels assimilables à l'arbitrage 'coutumier". Ce dernier consistait à soumettre un litige à un petit comité (la Cour des sages) ou à un chef de famille, de la tribu en vue d'aboutir à un compromis acceptable par les deux parties et dont les effets étaient contraignants. Cf. T. OLAWALE ELIAS, La nature du droit coutumier africain, Paris, 1961, pp. 231-232. Lire également, MBOG BASSOG, La théorie du droit en Afrique. Concept, objet, méthode et portée, Paris, 2016, pp. 196 et suiv. Lire aussi, J.-Y. LE BORGNE, Changer la justice, Paris, 2017, p. 130.

11 S. BEBOHI, "Les avantages comparatifs des Règlements d'arbitrage CIRDI-CNUDCI-CCJA", European Journal of Law Reform, 2011 (13), pp. 514-528, p. 516.

12 Signalons que l'arbitrage CCJA s'ouvre lorsqu'il y a, prima facie, une convention d'arbitrage visant l'application du Règlement d'arbitrage de la CCJA. En ce sens, le recours à l'arbitrage CCJA nécessite un lien de rettachement avec un Etat partie au Traité de l'OHADA. İl peut ainsi s'agir d'une personne(particuliers et personnes publiques) domiciliée ou résidant habituellement dans un 
Pour faire état de ces éléments d'influences mutuelles de la CCJA et l'EACJ, en vue d'une indépendance de la justice sur le continent africain, nous avons fait le choix d'une analyse en quatre temps articulée autour du concept central de nos assises à savoir l'indépendance de la justice.

Ainsi, en premier lieu, nous allons vérifier si dans pareille promotion de l'arbitrage, qui emporte changement d'esprit de la justice, l'indépendance judiciaire n'y perd pas son âme. Ainsi, sera analysée la portée de l'indépendance de la justice arbitrale encadrée par la CCJA (A). En deuxième et troisième lieux, nous mettrons en lumière les éléments d'influence des deux ordres arbitraux EACJ-CCJA en vue de renforcer l'indépendance des arbitres (B-C). On peut donc se limiter à ces quelques éléments, tout en montrant, en dernier lieu, qu'ils pourront constituer à l'avenir des bases de discussion sur lesquels pourront s'arrimer les possibilités d'emprunts mutuels entre la CCJA et l'EACJ (D).

\section{A. Le contenu normatif de l'indépendance de la justice arbitrale}

L'OHADA veut mener une transition judiciaire profonde en changeant les paradigmes de la justice étatique pour s'appuyer sur les MARC. Ces derniers sont en plein essor dans l'espace OHADA, qu'on ne cesse plus de vanter leurs mérites. Mais en réalité, quelles sont les vertus des MARCS de l'OHADA à enrayer l'insécurité judiciaire dans les Etats parties? En particulier, l'OHADA s'érige déjà en une valeur de référence sur le continent africain en matière d'arbitrage. A travers elle, les Etats se sont dotés d'un acte uniforme relatif au droit de l'arbitrage. Ce texte régit l'arbitrage ${ }^{13}$ de droit commun ${ }^{14}$. L'arbitrage institutionnel est régi par l'art. 2 et le titre IV du Traité de l'OHADA (art. 21-26) qui contient les principes de base. Le Règlement d'arbitrage de la CCJA $^{15}$, son Règlement intérieur règlent en détail le déroulement de l'arbitrage ${ }^{16}$. Toujours dans sa dynamique de contractualisation de la justice commerciale, l'OHADA vient de se doter d'un acte uniforme relatif à la médiation ${ }^{17}$. C'est surtout l'arbitrage institutionnel de la CCJA qui nous préoccupe ici.

Le Centre d'arbitrage de la CCJA/OHADA est chargé de la gestion des procédures d'arbitrages ouverts sous l'égide du Règlement d'arbitrage de la CCJA. C'est le Secrétaire

Etat partie ou d'un contrat eexécuté sur le territoire de l'un des Etats parties. Il n'est pas nécessaire que le siège de l'arbitrage soit situé dans un Etat partie.

13 Adopté le 11 mars 1999 (J.O. OHADA, 15 mai 1999) et révisé le 23 novembre 2017.

14 Cet acte uniforme, bien qu'étant le droit commun de l'abitrage dans l'espace OHADA, la CCJA lui a nié toute compétence de principe les textes qui regissent directement l'abitrage CCJA. Ainsi, dans son Arrêt $\mathrm{n}^{\circ} 44$ du 17 juillet 2008, la CCJA a retenu : «l'acte uniforme relatif au droit de l'arbitrage, ne figure pas au nombre des actes des actes juridiques précités qui sont applicables en l'espèce à l'arbitrage institutionnel spécifique de la C.C.J.A»

15 Règlement d'arbitrage de la Cour Commune de Justice et d'Arbitrage adopté le 11 mars 1999 (J.O. $O H A D A, 15$ mai 1999) et révisé le 23 novembre 2017.

16 P.-G. POUGOUE, L'arbitrage dans l'espace OHADA, Recueil des cours de l'Académie de droit international de La Haye, 2015, p. 130.

17 Adopté le 23 novembre 2017. 
Général qui sert d'interface entre les arbitres et la CCJA en assumant les attributions dont on se bornera à rappeler ici ${ }^{18}$. Le Président de la CCJA joue également un rôle important dans l'administration des procédures arbitrales ${ }^{19}$.

Parler de la normativité du principe de l'indépendance de la justice, revient à vérifier comment les règles qui régissent l'arbitrage institutionnel de la CCJA produisent un effet juridique, ou, carrément, sanctionnent le non-respect de l'indépendance de la justice. Cette indépendance peut se comprendre en termes de souveraineté des arbitres dans l'appréciation du fond de l'affaire ${ }^{20}$. L'indépendance de la justice arbitrale figure parmi les principes fondamentaux du droit international de l'arbitrage ${ }^{21}$. L'indépendance des arbitres fait partie des règles inaliénables dans le système arbitral de l'OHADA. L'indépendance de la justice arbitrale demeure au premier plan de la sécurité judiciaire tant recherchée par la CCJA ${ }^{22}$. Pour la garantie d'un procès équitable, la CCJA fait de l'indépendance des arbitres une exi-

18 En ce sens, le Secrétaire Général du centre d'arbitrage de la CCJA s'occupe notamment :

- De recevoir les demandes d'arbitrage de toute partie désirant avoir recours à l'arbitrage institué par l'article 21 du Traité OHADA ;

- Saisir la Cour pour la fixation de la provision pour les frais de l'arbitrage, pour la mise en œuvre de celui-ci, et s'il y a lieu, la fixation du lieu de l'arbitrage;

- Recevoir les mémoires, les correspondances et les notes écrites échangées par les parties, ainsi que toutes pièces annexes;

- Recueillir la copie du procès-verbal adressé aux parties et à leurs conseils;

- Recevoir toute demande en rectification d'erreurs matérielles d'une sentence, ou en interprétation de celle-ci, ou en complément de la sentence qui aurait omis de statuer sur une demande soumise à l'arbitre;

- Conserver dans les archives de la Cour toutes les sentences, le procès-verbal constatant l'objet de l'arbitrage et fixant le déroulement de la procédure, les décisions de la Cour, ainsi que la copie du courrier pertinent rédigé par le Secrétariat dans chaque affaire;

- Enregistrer sur un répertoire général toutes les affaires dont la Cour est saisie en matière d'arbitrage;

- Proposer pour adoption par la Cour, la nomenclature et le fonctionnement des différents comptes.

19 Cf. Règlement d'arbitrage CCJA, chapitre II.

20 J.-B. RACINE, "Réflexions sur les voies de recours en droit de l'arbitrage —Propos introductifs", Revue de l'Arbitrage, vol. 2018, Issue 1, pp. 3-13, p. 3.

21 Voir notamment le Règlement d'arbitrage de la CNUDCI, art. 11 ou encore le Règlement d'arbitrage du CIRDI, art. 6. Dans la pratique, des modèles-types de déclaration d'indépendance sont soumis à la signature des arbitres afin qu'ils signalent, avant leur nomination ou pendant l'instance arbitrale, tout fait ou toute circonstance de nature à compromettre leur indépendance ou leur impartialité.

22 En effet, l'insécurité judiciaire résulte d'une incertitude pesant sur l'issue de toute action en justice. La qualité du travail de la justice, dans les membres de l'OHADA, est très souvent contestée. Cela résulte de facteurs divers mais convergents, parmi lesquels on cite, à juste titre, le plus souvent, la saturation des juridictions, leurs moyens limités, le statut et la rémunération des magistrats et la nécessité de mettre à jour leur formation. Cette insécurité judiciaire se manifeste de façons très diverses : décisions contestables, décisions en délibéré depuis plusieurs années, exécutions impossibles, négligences diverses, méconnaissance des règles de déontologie, accueil des moyens dilatoires les plus évidents et renvois à répétition qui finissent par décourager les demandeurs de 
gence fondamentale. C'est pourquoi, malgré sa forte connotation éthique, elle a reçu l'onction du législateur du Règlement d'arbitrage de la CCJA qui l'a fait accéder à la scintillante juridicité à travers son article 4 . Cette règle permet de persuader les arbitres à ne pas entrer en contact avec des arbitrages dans lesquels ils seraient pressentis ${ }^{23}$.

Si l'on s'accorde sur les contours de ce principe d'indépendance, on peut maintenant envisager comment la CCJA et l'EACJ peuvent s'influencer mutuellement quant aux mesures qui visent à garantir l'indépendance des arbitres.

\section{B. Un processus de constitution du tribunal arbitral renforçant l'indépendance des arbitres CCJA}

Lors de la constitution du tribunal arbitral, le Centre d'arbitrage de la CCJA apporte son assistance en nommant, confirmant ou revoquant les arbitres. À ce stade, on donne priorité à la volonté des parties. Celles-ci se choisissent entre un ou trois arbitres. Et à défaut de choix par les parties, le tribunal arbitral sera composé des arbitres nommés par la CCJA. Mais cette désignation discrétionnaire de la Cour n'intervient que dans des circonstances exceptionnelles ${ }^{24}$. Ces arbitres sont choisis parmi les personnes figurant sur la liste des arbitres tenue par la CCJA. Ces personnes doivent justifier d'une expertise avérée en matière d'arbitrage international et peuvent être des ressortissants même des pays non membres à l'OHADA. Ces arbitres sont désignés en tenant compte essentiellement de leur indépen-

bonne foi... A noter que ces reproches peuvent aussi s'adresser à des auxiliaires de justice négligents, voire peu scrupuleux.

Dans un cas comme dans l'autre, l'harmonisation apparaît comme la solution à l'insécurité judiciaire; Mais les Etats n'ont pas pas abondonné leur autonomie institutionnelle à l'OHADA. Pour faire face à l'insécurité judiciaire, l'OHADA adopte les textes du droit harmonisé, qui abrogent les textes antérieurs contraires; ils opèrent ainsi un « toilettage » complet de leur dispositif de droit des affaires. Les effets pervers de textes désuets se trouvent ainsi écartés. Par ailleurs, ne pouvant pas se doter de tribunaux de première instance, elle s'assure de l'unité d'interprétation des actes uniformes par les juges nationaux en instaurant la CCJA, qui est une juridiction de cassation commune à tous les Etats parties. İl y a lieu de la comparer avec l'East African Court of Justice (EACJ) de l'EAC.

Mais en plus de cela, une bonne formation des magistrats doit être au cœur de la réponse que devra recevoir le problème de l'insécurité judiciaire, qu'il s'agisse de formation initiale ou de formation continue que l'OHADA assure à travers l'ERSUMA. İl reste à s'assurer que ce dispositif normatif et institutionnel parviendra à changer les suspicions et mépris quotidiens des acteurs de la justice. Cette pluralité mécanismes judiciaires permettent de protéger les opérateurs économiques de l'espace OHADA des incertitudes judiciaires.

23 Pareille conception de la normativité est partagée par S. GERRY-VERNIERES, Les " petites 》 sources du droit (A propos des sources étatiques non contraignantes), Thèse Paris II, 2010, $\mathrm{n}^{\circ} 316$, p. 172.

24 Règlement d'arbitrage CCJA à l'art. $3 \S 3.3$ "Si, pour quelque motif que ce soit, la nomination ne peut pas être faite en vertu de cette procédure, la Cour peut exercer son pouvoir discrétionnaire pour nommer un ou plusieurs arbitres". 
dance vis-à-vis des parties ${ }^{25}$. Contrairement à l'arbitrage EACJ dont les juges sont en même temps arbitres. Ce cumul des statuts des juges et arbitres du système arbitral de l'EACJ risque de compromettre leur indépendance. Rappelons que ces juges ne sont pas nommés en raison de leur expertise mais plutôt en représentation des Etats dont ils sont ressortissants. Les juges de l'EACJ sont proposés par leurs Etats dont ils dépendent en tant que magistrats ou fonctionnaires de l'appareil judiciaire national.

En effet, suivant l'article 32 (c) du Traité de l'EAC :

"The Court shall have jurisdiction to hear and determine any matter: ... (c) arising from an arbitration clause contained in a commercial contract or agreement in which the parties have conferred jurisdiction on the Court".

İl ressort de cette disposition que les investisseurs peuvent saisir directement l'EACJ d'une demande qui tire son origine d'un contrat commercial ou d'une clause compromissoire. C'est cette dernière qui nous intéresse ici. İl est d'une pratique courante pour les Etats de l'EAC de référer à l'arbitrage du $\mathrm{CIRDI}^{26}$ pour resoudre les différends les mettant aux prises avec les investisseurs étrangers. A cette possibilité s'ajoute désormais celle d'un arbitrage à assurer par la Cour de justice de l'EAC. Les conditions de déroulement de cette instance d'arbitrage au sein de la Cour de l'EAC sont déterminées dans the Arbitration Rules de 2012. Le déroulement emprunte les méandres habituels des arbitrages internationaux mais la désignation des arbitres et de la langue est loin d'être libérale.

L'Arbitration Rules reduit la sphère de l'autonomie de volonté des parties ${ }^{27}$ à l'abitrage EACJ notamment en leur imposant le choix des arbitres parmi les juges de 1 'EACJ ${ }^{28}$. Ces

25 D'autres facteurs expliquent que le choix soit porté sur un arbitre plutôt que sur un autre notamment la nationalité et le lieu de résidence des parties, le lieu de résidence des autres arbitres et conseils, l'objet du litige, le droit applicable et la langue des parties.

26 Suivant l'art. $1 \S 2$ de la Convention du 18 mars 1965 et le Règlement subsquent adopté en avril 2006, le CIRDI se charge d'assurer l'arbitrage des différends relatifs aux investissements opposant ses Etats membres à des ressortissants d'autres Etats membres.

27 L'autonomie de volonté fait partie des principes fondamentaux de l'abitrage. L'expression de cette volonté se manifeste notamment par la constitution du tribunal arbitral et le choix de la lagnue de l'arbitrage. On trouve ce principe affirmé aussi bien dans l'arbitrage CCJA (Règlement d'arbitrage CCJA, Art. 3), le système d'abitrage CIRDI (art. 1§ 1) que dans l'abitrage CNUDCI (Règlement de 2010, art. $3 \S 3$ ). L'EACJ semble ignorer encore ce principe en matière de choix de la langue de l'arbitrage. En effet, l'article 22 du Règlement d'arbitrage de la CJEAC impose l'anglais comme langue de tout arbitrage. Les rédacteurs du Arbitration Act ne devaient pas perdre de vue que l'abitrage EACJ a cours dans un environnement plurilingue dans lequel des centaines de langues nationales coexistent avec l'anglais et le français utilisés dans la communication judiciaire au sein des Etats membres. Aussi, que la langue de la justice arbitrale constitue l'un des éléments de son attractivité. İl est vrai que l'anglais est doté d'un statut dominant dans les pays membres de l'EAC mais imposer l'anglais comme langue unique de l'arbitrage reduirait l'attractivité de ce mécanisme et sera préjudiciable aux ressortissants du Burundi, par exemple, qui sont francophones. Lire à ce sujet, N. HALAOUI, "La langue de la Justice et les Consitutions africaines", Droit et société, 2002/2 (n'51-52), p. 345-367, p. 346.

28 Règlement d'arbitrage de la EACJ, art. 8. 
juges-arbitres sont désignés en raison de leur expertise afférente à l'affaire concernée et en tenant compte des impératifs d'indépendance et d'impartialité. L'arbitrage à assurer à ce niveau s'avère être un processus indépendant et impartial comparé aux instances qui seraient ouvertes au sein des juridictions étatiques que contrôlent les Etats membres de l'EAC. Toutefois, ces juges de l'EACJ qui demeurent attachés au service dans leurs Etats parties. Cela peut susciter des inquiétudes sur leur allégeance aux Etats dont ils sont ressortissants. Ce qui peut laisser subsister des doutes sur la souveraineté avec laquelle ils rendent leurs sentences ${ }^{29}$. On soupçonnerait dès lors les Etats de vouloir reprendre un contrôle indirect sur l'arbitrage dans cette région. Cela ne pourrait se comprendre, même pour les grandes transactions commerciales dans lesquelles l'intérêt général de l'Etat, si l'on se préoccupe pas des investisseurs considérés comme des parties économiquement faibles.

Les juges de la CCJA ne peuvent véritablement se prononcer sur l'arbitrage qu'une fois la sentence rendue en cas de recours en validité de la sentence ou en annulation de la sentence ${ }^{30}$ de révision ou encore de tierce opposition. Dans ce cas, la requête doit être déposée dans un délai de deux mois à compter de la notification de la sentence ${ }^{31}$.

29 Cf. Plan stratégique de la CJEAC, 2010-2015, p. 15.

30 Et dans ce cas, la CCJA peut également statuer au fond si les parties lui en ont fait la demande. Dans le cas contraire, la procédure d'arbitrage est reprise à la requête de la partie la plus diligente.

31 La CCJA a développé une jurisprudence énorme sur le contentieux de l'arbitrage. La CCJA s'est exprimée sur l'autonomie de la convention d'arbitrage, (cf. Arrêt n 097/2015 du 23 juillet 2015 dans l'affaire SODIMA SA devenue SANIA-Cie SA Contre DRAMERA Mamadou, Arrêt $\mathrm{n}$ ○082/2014 du 22 mai 2014 dans les affaires Société CANAC Sénégal S.A. et Société CANAC Railway Services Inc, Contre Société TRANSRAIL S.A.), le principe de la « compétence-compétence » des arbitres et du principe de la validité de la convention d'arbitrage qui doit s'apprécier, sans référence nécessaire à un droit étatique, donc d'après la commune volonté des parties (Arrêt $\mathrm{N}$ '069/2015 du 29 avril 2015 dans l'affaire La société Bougainvilliers, La société Immobilière Thiam Banda, devenue société d'investissements Thiam Banda SA, Les Héritiers de feu Mayoro Wade contre Monsieur Paul Mochet; Arrêt n 012/2011, 29 novembre 2011 dans l'affaire République de Guinée Equatoriale et la Communauté Economique et Monétaire de l"Afrique Centrale (CEMAC) contre la Commercial Bank Guinea Ecuatorial-CBGE), sur le respect du principe du contradictoire (Arrêt n ${ }^{\circ} 011 / 2015$ du 02 avril 2015 dans l'affaire Agence pour la Sécurité de la Navigation Aérienne en Afrique et à Madagascar dite ASECNA, contre Société SNACK BAGS; Arrêt n¹03/2015 du 15 octobre 2015 dans l'affaire Société Benin Control SA contre Etat du Bénin), le caractère limitatif de l'énumération des motifs d'annulation ou de cas d'ouverture de contestation de validité de sentence arbitrale (Arrêt $\mathrm{n}^{\circ}$ 062/2012 du 07 juin 2012 dans 1'affaire Société constructions métalliques ivoiriennes dite CMI contre Fraternité Saint Jean Eudes D'Abatta; Arrêt $\mathrm{n}^{\circ}$ 045/2008, 17 Juillet 2008 dans l'affaire Société Nationale pour la Promotion Agricole SONAPRA contre Société des Huileries du Bénin dite SHB; arrêt nº 043/2008, 17 juillet 2008 dans l'affaire Dam Sarr contre Mutuelle d'Assurance des Taxis Compteurs d'Abidjan dite MATCA), sur la compétence du juge étatique pour prendre des mesures conservatoires (Arrêt $n^{\circ} 018 / 2015$ du 02 avril 2015 dans l'affaire Société United Bank for Africa-UBA; Arrêt nº7/2015 du 27 avril 2015 dans l'affaire : Liquidation société CIM SAHEL ENERGIE contre Société « les Ciments du Sahel dite CDS S.A) Contre Société Beneficial Life Insurance-BLI), l'exclusion des voies de recours de droit commun contre une sentence arbitrale (Arrêt n ${ }^{\circ}$ 021/2015 du 09 avril 2015 dans l'affaire Société EMCICA Congo SARL contre Société ELECTRA SA), les motifs d'annulation des sentences notamment celui relatif à la violation de l'ordre public international (Arrêt n ${ }^{\circ} 104 / 2015 \mathrm{du}$ 
Mais au niveau de la constitution du tribunal arbitral, on peut s'interroger sur certaines mesures d'ordre préventif et curatif qui doivent être prises pour garantir l'indépendance de l'arbitre vis-à-vis des parties.

Suivant le Règlement d'arbitrage de la CCJA :

« Avant sa nomination ou sa confirmation par la Cour, l'arbitre pressenti, révèle par écrit au Secrétaire Général toutes circonstances de nature à soulever des doutes légitimes sur son impartialité ou son indépendance... L'arbitre fait connaître immédiatement par écrit au Secrétaire Général et aux parties, toutes circonstances de même nature qui surviendraient entre sa nomination ou sa confirmation par la Cour et la notification de la sentence finale $»^{32}$.

Il pèse sur l'arbitre une obligation d'information qu'on peut qualifier d'un « serment d'indépendance de l'arbitre ». Il semble qu'en pratique ces déclarations ne sont pas suivies d'investigations sérieuses ${ }^{33}$.

Afin d'éviter, autant que possible, que des pressions, ou des conflits d'intérêts n'entament l'issue de l'arbitrage, le Règlement d'arbitrage de la CCJA ouvre une possibilité de récusation des arbitres. Elle la complète par une possibilité de déport des juges de la CCJA appelés à connaître de l'examen de la sentence.

La récusation est encadrée par des délais et des procédures stricts. Ce dispositif permet à une partie d'écarter des arbitres suspectés de pression ou de lien compromettant pouvant influer sur l'issue de l'instance arbitrale. A l'état actuel de la jurisprudence arbitrale de la CCJA, nous n'avons pas trouvé d'affaires lesquelles les arbitres seraient récusés. Au passage, une décision de récusation ne représente qu'une simple mesure d'administration de l'instance arbitrale non susceptible de recours ${ }^{34}$. Étant remarqué, en passant, que leur nature administrative justifierait, sinon expliquerait, qu'elles ne soient pas alignées dans les différentes décisions arbitrales de la CCJA rendues conformément à ses attributions juridictionnelles ${ }^{35}$.

15 octobre 2015 dans l'affaire Etat du Bénin représenté par l'Agent Judiciaire du Trésor contre Société Commune de Participation (SCP) \& Patrice Talon; Arrêt n ${ }^{\circ} 03 / 2015$ du 15 octobre 2015 dans l'affaire Société Benin Control SA contre Etat du Bénin); Extension de la clause compromissoire (Arrêt n ${ }^{\circ}$ 12, 24 février 2005 dans l'affaire Société de manufacture de Côte d'Ivoire dite MACACI contre May Jean Pierre, Arrêt n ${ }^{\circ}$ 24, 08 avril 2010, dans l'affaire Joseph Roger cl Fofana), la litispendance arbitrale (arrêt n ${ }^{\circ}$ 47, 15 juillet 2010, dans l'affaire Société Navale Guinéenne dite SNG S.A. c/ Société Africaine de Commerce dite SAFRICOM).

32 Règlement d'arbitrage de la CCJA, art.4 §. 4.1 al. 3 et 5 .

33 P.-G. POUGOUE, op. cit., p. 210.

34 Règlement d'arbitrage de la CCJA, art. 1§ 1.1. lire aussi, P. MEYER, "Commentaire sous l'article 4 du Règlement d'arbitrage de la CCJA" in OHADA. Traité et acte uniformes commentés et annotés, Juriscope, 2012, p. 196.

35 Cette compétence s'exerce conformément au Chapitre III du Règlement d'arbitrage de la CCJA ainsi que du Règlement de procédure de la CCJA. 
Quoiqu'il en soit, la question s'était plutôt posée de manière hasardeuse en instance de contestation d'une sentence arbitrale devant les juges de la $\mathrm{CCJA}^{36}$. A l'époque, l'hypothèse de récusation des juges de la CCJA n'était pas encore envisagée par son Règlement d'arbitrage (était-ce pour contrer l'éventualité des manœuvres dilatoires?). La révision de ce Règlement le 23 novembre 2017, sans viser directement la récusation des juges de la CCJA, s'est plutôt penchée sur l'hypothèse de déport. Ce dispositif suppose une auto-récusation à l'initiative du juge pressenti ${ }^{37}$. Il s'agit d'une évolution par rapport aux pratiques antérieures qui ont admis par exemple qu'un juge de la CCJA siégeant, au mépris du principe universellement reconnu en arbitrage international nemo index in causa sua, en arbitrage impliquant l'Etat de Guinée dont il est ressortissant ${ }^{38}$. L'impartialité de ce juge a d'ailleurs fait l'objet de polémique à la suite de l'annulation de la sentence Getma contre la République de Guinée comme vu plus haut.

Le législateur du Règlement d'arbitrage révisé de la CCJA ne limite ce déport qu'au cas de l'existence d'un lien entre un juge de la Cour avec un Etat partie à une instance arbitrale $^{39}$. Dans d'autres cas, explicitement, en dehors des arbitrages d'investissement ${ }^{40}$, ce déport n'est pas envisageable moins encore la récusation dont les parties ne pourraient se prévaloir. On peut légitimement s'interroger si la CCJA s'assure des mesures de déontologie de ses juges qui les mettraient à l'abri de tout pressentiment. Ce questionnement pessimiste est demeuré irrémédiable à l'issue de la révision du Règlement d'arbitrage de la CCJA intervenue fin 2017, ce ne peut être que partie remise.

36 Dans cette affaire, il avait été introduit une requête en récusation de tous les juges de la Cour. Faute de preuve et en l'absence d'un régime de récusation dans le Règlement de procédure de la CCJA. La Cour y a répondu en des termes qui méritent d'être cités : "... Attendu que cette requête en récusation ne repose sur aucun fondement juridique tel que l'a reconnu elle-même la partie demanderesse à l'occasion de sa plaidoirie à l'audience; attendu qu'en outre, l'Etat du Bénin ne rapporte, à l'appui de sa requête, aucune preuve des faits qu'il allègue et n'offre de le faire; mais attendu qu'il ne résulte ni du Traité constitutif de l'OHADA, ni du Règlement de procédure de la cour de céans la possibilité de récusation d'un juge, a fortiori de tous les juges composant ladite juridiction communautaire; qu'il échet dès lors, en application dudit Traité et du Règlement de procédure, de déclarer irrecevable une telle demande qui n'a d'autre objectif manifeste qu'un déni de justice...”.cf. Arrêt nº84/2015 du 18 juin 2015 dans l'affaire Etat du Bénin contre Société Bénin Control SA \& Autres.

37 A. RUBBENS, Le droit judiciaire congolais, Tome II, Kinshasa, 1976, p. 79.

38 CCJA, Arrêt du 19 novembre 2015 portant annulation de la sentence du 29 avril 2014.

39 Cf. art. 1 § 1.1 al. 4 : "Les membres de la Cour ayant la nationalité d'un Etat impliqué directement dans une procédure arbitrale doivent se déporter de la formation de la Cour dans l'affaire en cause. Le Président de la Cour procède à leur remplacement, le cas échéant, par ordonnance".

40 C'est-à-dire "l'arbitrage qui oppose un ou plusieurs investisseurs, ressortissants d'un ou plusieurs Etats donnés, à l'Etat d'accueil de leurs investissements qui sont, pour celui-ci, des investissements étrangers". Cf. L. Ch. DELANOY et T. PORTWOOD, "La responsabilité de l'Etat pour déni de justice dans l'arbitrage d'investissement", Revue de l'Arbitrage, Vol. 2005, Issue 3, pp. 603-643, p. 603. 
L'arbitre peut également démissionner s'il a des justes motifs ${ }^{41}$. Un arbitre récusé ou qui a démissionné ou encore qui est décédé en cours d'instance, doit être remplacé 42 .

Tout compte fait, la pratique de l'arbitrage de l'EACJ n'est pas trop développée ${ }^{43}$ bien que les parties soient dispensées de payer des honoraires aux arbitres ${ }^{44}$. La voie d'arbitrage est d'ailleurs des fois fermées aux investisseurs étrangers dans certains pays de l'EAC. İl en est ainsi du Rwanda et du Burundi lorsqu'il y a eu un désaccord sur la constitution du tribunal arbitral avec l'investisseur ${ }^{45}$.

\section{Un barème des frais et honoraires qui ne favorise pas l'indépendance des arbitres CCJA comparé à la gratuité de l'arbitrage EACJ}

Dans le système arbitral CCJA, la saisine du tribunal arbitral est subordonnée notamment à la consignation et la provision des frais et honoraires d'arbitrage.

Aussi bien les frais de consignation pour la demande d'arbitrage que la provision pour les frais d'arbitrage sont versés au Secrétariat général de la CCJA ${ }^{46}$. Hormis les honoraires des avocats, ces frais administratifs et honoraires comprennent notamment les honoraires de l'arbitre, les frais administratifs, les frais éventuels de l'arbitre, les frais de fonctionnement du tribunal arbitral, les honoraires et frais des experts en cas d'expertise ${ }^{47}$. Tous ces frais et honoraires font partie des préalables pour la demande d'arbitrage et doivent être réglés avant la remise du dossier à l'arbitre ${ }^{48}$. Une demande de provision non satisfaite peut entraîner la suspension de l'instance ${ }^{49}$. Bien plus, après la réunion de cadrage, les frais peuvent être revus à la hausse en cas de demande reconventionnelle ${ }^{50}$.

41 Cette faculté de démissionner est strictement surveillée pour éviter une éventuelle complicité entre les arbitres et une partie.

42 Le remplacement est également possible pour tout autre empêchement notamment si la CCJA constate que l'arbitre est empêché de jure ou de facto de continuer sa mission, ou qu'il ne remplit pas ses obligations correctement.

43 Moins de 10 arbitrages jusque là.

44 Arbitration Rules (2012), art. 37.

45 Cf. Code des investissements du Rwanda, art. 34; Code des investissements de l'Ouganda, Sect. 28(4).

46 Parmi les conditions pour déposer la demande d'arbitrage à la CCJA, il est fait obligation à la requérante de consigner la somme équivalent à 200.000 Francs CFA.

47 Cf. les barèmes des frais d'arbitrage du Secrétariat Général de la CCJA (décision n0094/99/CCJA du 3 février 1999, J.O. OHADA, 15 mai 1999, p.23).

48 Règlement d'arbitrage CCJA, art. 8 et 11. Lire J. WAMBO, «L'Arbitrage CCJA », Revue de l'ERSUMA-Droit des affaires - Pratique Professionnelle, $\mathrm{n}^{\circ}$ Spécial - Nov-Déc 2011, Etudes. Lire aussi, J. M'BOSSO, "Le fonctionnement du Centre d'arbitrage CCJA et le déroulement de la procédure arbitrale", Revue camerounaise de l'arbitrage, numéro spécial, octobre 2001, pp. 42-52.

49 Règlement d'arbitrage CCJA, art. 11 in fine.

50 P.-G. POUGOUE, op. cit., p. 208. 
En règle générale, ces frais sont souvent très élevés comparés aux frais et droits de justice applicables dans les Etats membres ${ }^{51}$.

Ces frais ne sont pas à la disposition des arbitres. Les arbitres et les parties, ne peuvent pas, par accord exprès en modifier le régime. L'effacement délibéré de la présence des arbitres dans cette opération de paiement est un gage de leur indépendance vis-à-vis des parties. Mais l'arbitrage EACJ renforce encore plus l'indépendance des arbitres en prévoyant le principe de sa gratuité.

A une époque récente de l'arbitrage CCJA, cette question des frais a soulevé, quelles que soient les étiquettes, des polémiques sur l'indépendance de cette institution. Sur le fond du problème, les arbitres sont entrés en contact direct avec les parties pour négocier le montant de leurs honoraires au mépris des dispositions sus-citées du Règlement de la CCJA ${ }^{52}$. La CCJA a annulé leur sentence pour « la violation de leur mission » par les trois arbitres. Selon la Cour, « le tribunal arbitral ne s'est pas conformé à sa mission », " en écartant délibérément des dispositions essentielles du Règlement d'arbitrage » essentiellement celles des art. 8 et 11 qui prévoit que c'est le Secrétaire Général qui saisit la Cour pour la fixation ou le réajustement de la provision pour les frais d'abitrage. La Cour reproche notamment au tribunal arbitral le caractère immoral de cette transaction ${ }^{53}$ et réitère « les honoraires des arbitres sont exclusivement fixés par la Cour» et que cette dernière a rejeté par deux fois (1er août 2013 et 3 octobre 2013) la demande de relèvement des honoraires de 40,5 millions de F CFA à 295,2 millions de FCFA (soit 450000 euros).

Dans une sentence de la CCI, pour le cas tranché, on acceptait cette façon de faire litigieuse. En se fiant à l'apparence légale de cette transaction, la CCI a retenu une solution choquante méconnaissant malveillamment le droit de la CCJA d'annuler la sentence arbitrale rendue contrairement à son Règlement. L'argument semble plus dominé de fait que du droit. Il nous semble qu'en annulant cette sentence, la CCJA a procédé au contrôle de la manière dont les arbitres l'ont rendue ${ }^{54}$. En l'espèce, en se passant du régime d'ordre public de la fixation de leurs honoraires tel que prévu par le Règlement d'arbitrage de la CCJA leur sentence encourrait l'annulation. La CCI semble bouleverser le droit positif de l'arbitrage CCJA en admettant une contractualisation des honoraires entre arbitres et parties. Même après la révision du Règlement d'arbitrage de la CCJA, la liberté accordée pour revoir à la hausse les frais et honoraires ne le sont que dans d'étroites limites et là encore on ne permet pas aux arbitres d'entrer directement en contact avec les parties dans la fixation de leurs honoraires. Est-ce une « guerre » de règlements d'arbitrages qui s'annonce? La CCI tenterait de vendre le sien au marché subsaharien, qu'elle domine d'ailleurs à plus de

51 A. DİALO, Réflexion sur l'arbitrage dans l'espace OHADA, Thèse de doctorat, Faculté de Droit de l'Université de Perpignan, 2016, p. 220.

52 A la formation du tribunal arbitral, ces honoraires étaient fixés à 60.000 euros. Mais les arbitres les estimant dérisoires ont obtenu des parties que ces honoraires soient élevés à 450. 000 euros.

53 Le fait d'avoir obtenu le paiement par l'une des parties de 450000 euros au titre des honoraires des arbitres.

54 J.-B. RACINE, op. cit., p. 4. 
$75 \%$, et la CCJA qui voudrait affirmer son identité malgré la paternité de la CCI qu'elle ne sait renier?

\section{Conclusions}

Pour une comparaison rapide, instinctive, de caractère exploratoire, nous avons passé en revue les éléments de rapprochement entre la CCJA et l'EACJ en vue d'une justice arbitrale indépendante. Il était sans intérêt pour la présente communication d'aborder tous les aspects de la procédure arbitrale. Ainsi, elle ne rend pas compte les arbitrages CCJA et EACJ depuis la clause arbitrale jusqu'à l'exécution de la sentence.

La diversité du vocabulaire utilisé par les deux systèmes d'arbitrage ne nous a pas empeché de discerner des éléments utiles à puiser mutuellement par un système dans l'autre et inversement.

İl en est ainsi de la constitution du tribunal arbitral, dont il a été question plus haut. Ici, la pratique arbitrale CCJA pourrait être amenée à développer et à préciser les acquis du Règlement révisé notamment sur la notion du déport des juges de la CCJA qu'on pourrait alors assimiler au régime de la récusation des juges de la CCJA qui examinent la sentence.

Ici encore, dans l'arbitrage CCJA, l'arbitre est en principe délié de toute allégeance étatique alors que dans le système de l'EACJ, les juges étant toujours en poste de manière permanente dans leurs Etats d'origine, peuvent subir leur influence. Or, on sait que les Etats d'accueil ne sont pas toujours tendres dans leurs engagements avec les investisseurs ( $a$ priori parties en faiblesse économique et qui courre le danger de l'influence étatique) et que l'allégeance gardée entre l'arbitre-juge EACJ et son Etat entamerait son indépendance et, par ricochet, la crédibilité même du système arbitral EACJ. L'accord CETA entre 1'Union Européenne et le Canada, tend à reformer l'arbitrage d'investissement en suivant cet exemple des juges-arbitres en cours devant l'EACJ. La raison annoncée est de doter ce système arbitral de plus de légitimité. Mais, il nous semble que l'impératif "promotionnel" de légitimité ne saurait être poursuivi au mepris de l'indépendance et de l'impartialité des décisions à rendre. Les deux impératifs se recoupent car l'indépendance renforce la confiance placée dans les arbitres et garantit, par là même, la légitimité de tout le système arbitral. On est porté à penser que la pratique de l'arbitrage au sein de l'espace OHADA peut être inspirante pour l'EACJ.

L'arbitrage EACJ risque de tomber dans les travers du passé de la CCJA qui annulait des sentences arbitrales en comptant dans sa composition des juges ressortissants de l'Etat mis en cause. Agir comme juge judiciaire et arbitral à la fois relève d'une ambiguïté!!! Nous avons vu, sans qu'il ne soit nécessaire d'y revenir ici, que la CCJA fait l'objet de critiques acerbes sur son hybridité avancée. Cette nature la conduit à agir à la fois comme juge 
judiciaire et juge encadrant. La dissociation de ces deux casquettes serait une voie pour le renforcement de l'indépendance et l'impartialité des arbitrages que la CCJA supervise ${ }^{55}$.

Par ailleurs, sur le terrain des frais et honoraires à payer pour la procédure arbitrale, on pourrait émettre le souhait, sans plaider pour la gratuité de l'arbitrage, que les frais et honoraires soient stipulés de manière à permettre la maîtrise des coûts par les parties ${ }^{56}$. Dans le contexte de l'OHADA, l'arbitrage est perçu dans le prolongement des services publics de la justice des Etats parties ${ }^{57}$. L'OHADA devait permettre aux petites unités économiques ainsi qu'aux particuliers, frustrés par la justice étatique, de recourir à l'arbitrage à un moindre coût. On approuve donc, de ce point de vue, l'approche adoptée par l'EACJ dans la gratuité de la justice arbitrale pour plus d'indépendance des arbitres envers les parties. Sur le plan pratique, cette opinion risque de heurter la résistance de la CCJA mais ce ne peut être, en bonne logique, qu'inscrire l'arbitrage CCJA dans l'ordre marchand au mépris des considérations extra-économique d'accès à la justice arbitrale censées offrir une alternative à l'insécurité judiciaire dans l'espace OHADA ${ }^{58}$. Il est temps que 1'OHADA rende plus effectif le Fonds de promotion de l'arbitrage de l'OHADA, institué par la décision n ${ }^{\circ} 01 / 2012 / \mathrm{CM} /$ OHADA du 15 juin 2012. Ces fonds permettront d'assurer une assistance financière aux parties à l'arbitrage CCJA voire la promotion de tout arbitrage qui a lieu dans l'espace OHADA $^{59}$.

\section{REFERENCES BIBILIOGRAPHIQUES}

\section{Sources légales et conventionnelles}

Traité créant l'Organisation pour l'Harmonisation en Afrique du Droit des Affaires (OHADA) du 17 octobre 1993 tel que révisé le 17 octobre 2008.

Traité de l'EAC, 2001.

Code des investissements du Rwanda, 2005

Code des investissements de l'Ouganda,

Décision n0094/99/CCJA du 3 février 1999, J.O. OHADA, 15 mai 1999, p.23.

Règlement d'arbitrage de la CCJA adopté le 11 mars 1999 (J.O. OHADA, 15 mai 1999) et révisé le 23 novembre 2017.

55 Lire à cet effet, https://www.jeuneafrique.com/mag/292443/economie/guinee-honoraires-coutent-tr es-cher-a-getma/.

56 P.-G. POUGOUE, op. cit., p. 208.

57 Sans dénaturer l'arbitrage, on songe aux "valeurs de l'humanisme" assignées à l'arbitrage. $B$. OPPETIT, Théorie de l'arbitrage, Paris, PUF, 1988, p. 127.

58 Dans le même registre, un auteur s'est interrogé : "le coût élevé d'un arbitrage international constitue-t-il un obstacle à l'accès d'une partie faible à la Justice?". Cf. F. VALENCIA, "Parties faibles et accès à la justice en matière d'arbitrage", Revue de l'Arbitrage, 2007, Vol. 2007, Issue 1, pp. 4570. Lire aussi dans ce sens, M. SALAH, L'irruption des droits de l'homme dans l'ordre économique international, Mythe ou réalité?, LGDJ, Droit et Economie, Paris, 2012, p. 182 et ss.

59 P.-G. POUGOUE, op. cit., p. 209. 


\section{Jurisprudence}

Arrêt du 19 novembre 2015 portant annulation de la sentence du 29 avril 2014

Arrêt n 012/2011, 29 novembre 2011 dans l'affaire République de Guinée Equatoriale et la Coтmunauté Economique et Monétaire de l"Afrique Centrale (CEMAC) contre la Commercial Bank Guinea Ecuatorial-CBGE)

Arrêt n ${ }^{\circ}$ 021/2015 du 09 avril 2015 dans l'affaire Société EMCICA Congo SARL contre Société ELECTRA SA

Arrêt n ${ }^{\circ}$ 043/2008, 17 juillet 2008 dans l'affaire Dam Sarr contre Mutuelle d'Assurance des Taxis Compteurs d'Abidjan dite MATCA)

Arrêt n ${ }^{\circ}$ 045/2008, 17 Juillet 2008 dans l'affaire Société Nationale pour la Promotion Agricole SONAPRA contre Société des Huileries du Bénin dite SHB

Arrêt n ${ }^{\circ}$ 062/2012 du 07 juin 2012 dans l'affaire Société constructions métalliques ivoiriennes dite CMI contre Fraternité Saint Jean Eudes D'Abatta

Arrêt n ${ }^{\circ}$ 097/2015 du 23 juillet 2015 dans l'affaire SODIMA SA devenue SANIA-Cie SA Contre DRAMERA Mamadou

Arrêt n ${ }^{\circ}$ 24, 08 avril 2010, dans l'affaire Joseph Roger contre Fofana

Arrêt n 47, 15 juillet 2010, dans l'affaire Société Navale Guinéenne dite SNG S.A. contre Société Africaine de Commerce dite SAFRICOM

Arrêt n ${ }^{\circ} 011 / 2015$ du 02 avril 2015 dans l'affaire Agence pour la Sécurité de la Navigation Aérienne en Afrique et à Madagascar dite ASECNA, contre Société SNACK BAGS

Arrêt n ${ }^{\circ}$ 018/2015 du 02 avril 2015 dans l'affaire Société United Bank for Africa-UBA

Arrêt nº69/2015 du 29 avril 2015 dans l'affaire La société Bougainvilliers, La société Immobilière Thiam Banda, devenue société d'investissements Thiam Banda SA, Les Héritiers de feu Mayoro Wade contre Monsieur Paul Mochet

Arrêt nº82/2014 du 22 mai 2014 dans les affaires Société CANAC Sénégal S.A. et Société CANAC Railway Services Inc, Contre Société TRANSRAIL S.A.

Arrêt nº84/2015 du 18 juin 2015 dans l'affaire Etat du Bénin C/ Sté Bénin Control SA \& Autres.

Arrêt n ${ }^{\circ} 03 / 2015$ du 15 octobre 2015 dans l'affaire Société Benin Control SA contre Etat du Bénin

Arrêt n 104/2015 du 15 octobre 2015 dans l'affaire Etat du Bénin représenté par l'Agent Judiciaire du Trésor contre Société Commune de Participation (SCP) \& Patrice Talon

Arrêt n ${ }^{\circ}$ 12, 24 février 2005 dans l'affaire Société de manufacture de Côte d'Ivoire dite MACACI contre May Jean Pierre

Arrêt nº7/2015 du 27 avril 2015 dans l'affaire Liquidation société CIM SAHEL ENERGIE contre Société « les Ciments du Sahel dite CDS S.A) Contre Société Beneficial Life Insurance-BLI)

\section{Ouvrages et articles}

A. DİALLO, Réflexion sur l'arbitrage dans l'espace OHADA, Thèse de doctorat, Faculté de Droit de l’Université de Perpignan, 2016. 
A. RUBBENS, Le droit judiciaire congolais, Tome II, Kinshasa, Presses universitaires du Congo, 1976, p. 79

B. OPPETIT, Théorie de l'arbitrage, Paris, PUF, 1988.

F. VALENCİA, "Parties faibles et accès à la justice en matière d'arbitrage", Revue de l'Arbitrage, 2007, Vol. 2007, Issue 1, pp. 45-70.

J. M'BOSSO, "Le fonctionnement du Centre d'arbitrage CCJA et le déroulement de la procédure arbitrale", Revue camerounaise de l'arbitrage, numéro spécial, octobre 2001, pp. 42-52.

J. WAMBO, «L'Arbitrage CCJA », Revue de l'ERSUMA-Droit des affaires-Pratique Professionnelle, $\mathrm{n}^{\circ}$ spécial, novembre-décembre, 2011.

J.-B. RACINE, "Réflexions sur les voies de recours en droit de l'arbitrage —Propos introductifs", Revue de l'Arbitrage, vol. 2018, Issue 1, pp. 3-13.

J.-Y. LE BORGNE, Changer la justice, Paris, PUF, 2017.

L. Ch. DELANOY et T. PORTWOOD, "La responsabilité de l'Etat pour déni de justice dans l'arbitrage d'investissement", Revue de l'Arbitrage, Vol. 2005, Issue 3, pp. 603-643.

M. BASSOG, La théorie du droit en Afrique. Concept, objet, méthode et portée, Paris, MeduNeter, 2016.

M. SALAH, L'irruption des droits de l'homme dans l'ordre économique international, Mythe ou réalité?, LGDJ, Droit et Economie, Paris, 2012.

N. HALAOUI, "La langue de la Justice et les Consitutions africaines", Droit et société, 2002/2 (n $\left.{ }^{\circ} 51-52\right)$, p. 345-367.

P. MEYER, "Commentaire sous l'article 4 du Règlement d'arbitrage de la CCJA" in OHADA. Traité et acte uniformes commentés et annotés, Juriscope, 2012.

P.-G. POUGOUE, L'arbitrage dans l'espace OHADA, Recueil des cours de l'Académie de droit international de La Haye, 2015.

S. BEBOHI, "Les avantages comparatifs des Règlements d'arbitrage CIRDI-CNUDCI-CCJA", European Journal of Law Reform, 2011 (13), pp. 514-528.

S. GERRY-VERNIERES, Les " petites » sources du droit (A propos des sources étatiques non contraignantes), Thèse Paris II, 2010.

T. OLAWALE ELIAS, La nature du droit coutumier africain, Paris, Présence africaine, 1961. 